\title{
SUSY searches with two opposite-sign same-flavor leptons at CMS
}

\section{Sergio Sánchez Cruz*}

On behalf of the CMS Collaboration

Universidad de Oviedo (ES)

E-mail: sergio.sanchez.cruzecern.ch

A search is presented for physics beyond the standard model in final states with two oppositesign same-flavor leptons (electrons or muons), jets, and missing transverse momentum. The data sample corresponds to an integrated luminosity of $12.9 \mathrm{fb}^{-1}$ of proton-proton collisions at $\sqrt{s}=13$ TeV collected with the CMS detector at the CERN LHC during 2016. The analysis focuses on a search region with events containing leptons consistent with the decay of a $\mathrm{Z}$ boson, developing a data-driven method for the estimation of the Z+jets background based on the imbalance between the $\mathrm{Z}$ candidate and the recoiling jets. The observations in the signal region are consistent with the standard model expectations.

38th International Conference on High Energy Physics 3-10 August 2016

Chicago, USA

${ }^{*}$ Speaker. 


\section{Introduction}

This document reports a search for new physics performed in events with two opposite-sign, same-flavour leptons, compatible with the decay of a $\mathrm{Z}$ boson. Selected events are also required to contain additional jets and missing transverse momentum. These searches are motivated by SUSY GMSB models involving the production of an on-shell $\mathrm{Z}$ boson in the decay of a neutralino, as it is shown in fig. 1.

The ATLAS Collaboration reported a $3 \sigma$ excess [3] and a $2.2 \sigma$ excess [4] in searches for this topology using run I and run II data respectively. The CMS Collaboration however reported results compatible with SM expectations [2]. The results shown in this document are obtained with a dataset of $12.9 \mathrm{fb}^{-1}$ of $\sqrt{s}=13 \mathrm{TeV}$ pp collisions recorded by CMS during 2016 and are fully documented, in the context of a more complete analysis, in [1].

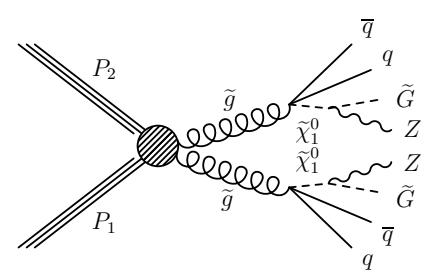

Figure 1: Diagram for gluino-gluino production, decaying into two quarks and a neutralino, which emits a $\mathrm{Z}$ boson in its decay into a gravitino.

\section{Search strategy}

The analysis focuses in events with two opposite-sign, same-flavour leptons. Additional requirements are imposed on the missing transverse momentum and the jet multiplicity. The spectrum of the dilepton invariant mass is splitted into a on- $Z$ part, in which the mass is compatible with that of the $\mathrm{Z}$ boson, and two another regions with masses above or below the $\mathrm{Z}$ boson. In this document, we focus on the on- $Z$ region, where the main background comes from $Z+j$ ets processes. While this latter background was predicted using the $E_{\mathrm{T}}^{\text {miss }}$ templates, here we present a different approach, using the Jet-Z Balance (JZB) method.

In particular, we perform the search in the region in which the excess was observed. This region is defined as events with two leptons with $p_{\mathrm{T}}>50(25) \mathrm{GeV}$, at least two jets with $p_{\mathrm{T}}>35$ $\mathrm{GeV}$ and $\Delta \phi_{\text {jet }, E_{\mathrm{T}}^{\text {miss }}}>0.4$.

\section{SM background estimation}

The Standard Model backgrounds contributing to the search region are flavour-symmetric processes, for which it is equally probable to have an opposite-flavour dilepton pair as a same-flavour dilepton pair (mainly $t \bar{t}$ production), and $\mathrm{Z}+$ jets events. The contribution of these processes to the search region is estimated using fully data-driven methods, while minor contributions from other SM processes are obtained from Monte Carlo simulations. 


\subsection{Flavour-symmetric backgrounds}

The contribution of flavour-symmetric processes is dominated by $t \bar{t}$ pair production. These processes are estimated taking the opposite-flavour channel as a side-band, correcting by the different trigger, reconstruction and identification efficiencies by a factor $r_{S F / O F}$, which is obtained from control regions in collision data, as it is reported in [1].

\subsection{Jet-Z Balance method}

The JZB, defined as JZB $=\left|p_{\mathrm{T}}^{\ell \ell}\right|-\left|\sum \vec{p}_{\mathrm{T}}^{\mathrm{jet}}\right|$, is a measure of the momentum unbalance of an event. As it is seen in fig. 2, it is symmetrically distributed for $Z+$ jets events, while for events with invisible particles in the final state (t $\bar{t}$ and signal), its distribution is tilted to positive values. In the JZB method, signal regions are defined with positive values of JZB, using the negative part of the distribution to estimate the $\mathrm{Z}+\mathrm{jets}$ contribution. A subtraction of the opposite-flavour channel corrected by the $r_{S F / O F}$ factor is performed to this estimation in order to avoid double counting of flavour-symmetric processes with JZB $<0$.

Figure 2 (center) shows the symmetry of the JZB distribution on Z+jets Monte Carlo simulations, and a $30 \%$ of systematic uncertainty is assigned to cover the small fluctuations observed in the tails, which are due to the small number of simulated events in those regions. The full closure of all the data-driven methods performed with Monte Carlo simulations is shown to have a clear agreement within systematic uncertainties in fig. 2 (right).
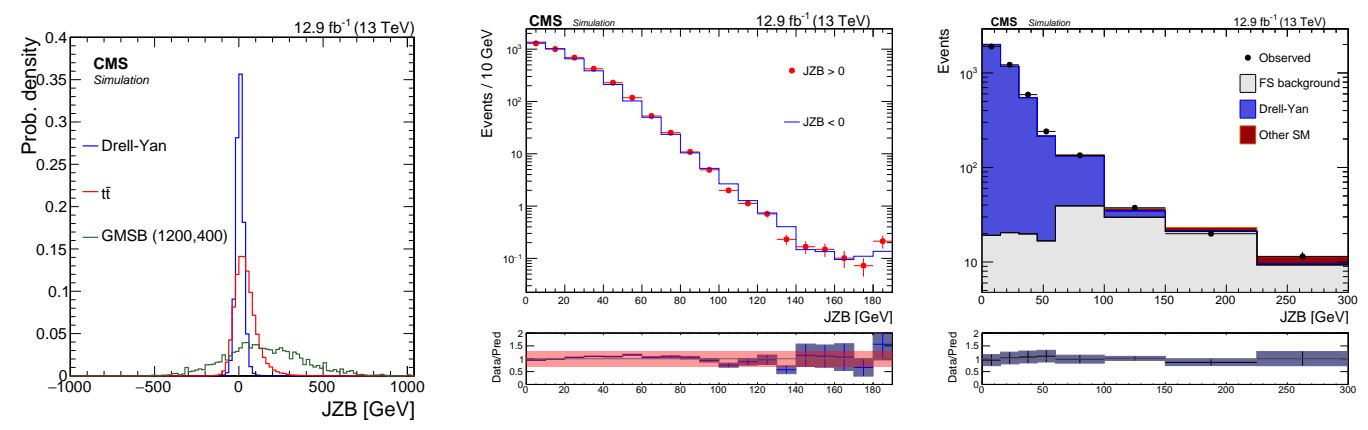

Figure 2: (Left) Distribution of the JZB variable in $Z+j e t s, t \bar{t}$ and signal simulated events, (center) symmetry of the JZB distribution in Z+jets simulated events and (right) full closure test of the JZB method in simulations.

\section{Results}

The results of the application of the JZB method to the excess region is shown in fig. 3 , in which the JZB distribution of the events observed in that region is shown, together with the estimation of the SM backgrounds provided by the different estimation methods. Table 1 displays the number of observed events in the region with JZB $>225 \mathrm{GeV}$ together with its estimation based on SM expectations. The results obtained using the $E_{\mathrm{T}}^{\text {miss }}$ templates method are also shown in fig. 3 .

The results shown agree with SM expectations, not showing the deviation observed by the ATLAS Collaboration. Therefore, no evidence for BSM physics can be inferred from these results in this analysis. 


\begin{tabular}{ll}
\hline FS estimate & $4.4_{-2.1}^{+3.5}$ \\
Drell-Yan like & $0.0_{-0}^{+1.8}$ \\
Other SM & $1.7_{-0.8}^{+0.8}$ \\
\hline Total background & $6.1_{-2.2}^{+4.0}$ \\
\hline Observed & 8.0 \\
\hline
\end{tabular}

Table 1: Observed events with JZB $>225 \mathrm{GeV}$
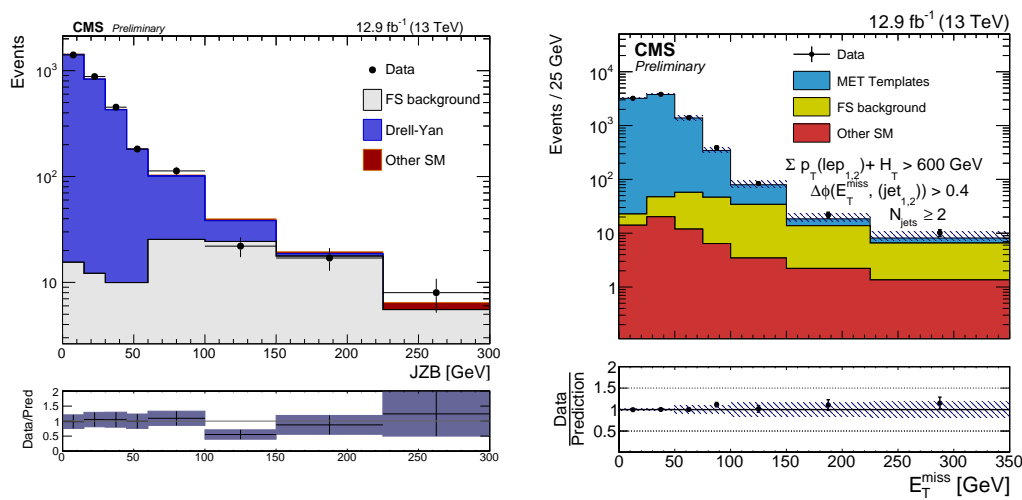

Figure 3: JZB (left) and $E_{\mathrm{T}}^{\text {miss }}$ (right) distributions distributions in the signal region determined using the JZB method and the $E_{\mathrm{T}}^{\text {miss }}$ templates method, respectively.

\section{Conclusions}

A search for new physics is presented in events with two opposite-sign, same-flavour leptons compatible with the decay of a $\mathrm{Z}$ boson, additional jets and missing transverse momentum using the JZB method to predict the contribution of the Z+jets background. Events were also required to be in a region in which an excess was observed by the ATLAS Collaboration in runs I and II. Results are found to be compatible with SM expectations.

\section{References}

[1] CMS Collaboration, "Search for new physics in final states with two opposite-sign, same-flavor leptons, jets, and missing transverse momentum in pp collisions at sqrt s $=13 \mathrm{TeV}$ ",

CMS-PAS-SUS-16-021.

[2] CMS Collaboration, "Search for new physics in final states with two opposite-sign, same-flavor leptons, jets, and missing transverse momentum in pp collisions at $\sqrt{s}=13 \mathrm{TeV}$ ", arXiv:1607.00915 .

[3] ATLAS Collaboration, "Search for supersymmetry in events containing a same-flavour opposite-sign dilepton pair, jets, and large missing transverse momentum in $\sqrt{s}=8 \mathrm{TeV}$ pp collisions with the ATLAS detector", Eur. Phys. J. C 75, no. 7, 318 (2015)

[4] ATLAS Collaboration, "A search for Supersymmetry in events containing a leptonically decaying $Z$ boson, jets and missing transverse momentum in $\sqrt{s}=13 \mathrm{TeV} p p$ collisions with the ATLAS detector", ATLAS-CONF-2015-082. 